\title{
Correspondence
}

To the Editors:

\section{Paracetamol in children}

Sri Lanka Journal of Child Health, 2003; 32: 83

(Key words: paracetamol, children)

The insert that appeared in the last issue of the Sri Lanka Journal of Child Health gives an incorrect sense of comfort to the users of paracetamol (acetaminophen). Paracetamol has been in use for the past 50-60 years and ever since aspirin was found to be a causative agent for Reye syndrome in 1980 s, paracetamol has come to stay as the drug of first choice for fever in children.

Firstly, children rarely ingest paracetamol in high doses. Many reported cases of severe hepatotoxicity in children have been attributed to cumulative toxicity from repeated doses rather than acute intoxication from a single massive overdose ${ }^{1}$. The general public should be educated regarding the problems with daily intake of supra-therapeutic doses for treatment of a febrile illness rather than the safety profile of a single acute overdose.

Secondly, recent reviews identified several factors associated with paracetamol hepatotoxicity in children including, age $<10$ years associated with inappropriate dosing, delay in onset of symptoms after a potentially toxic ingestion, delay in initiation of $\mathrm{N}$-acetyl cysteine treatment, unintentional multiple overdosing, ingestion of paracetamol along with another hepatotoxic drug ${ }^{1}$ and use of adult rather than paediatric preparations ${ }^{2}$. Failure to read and understand instructions on the label or use of an incorrect measuring device or preparation were cited as the usual causes of unintentional overdosing ${ }^{1}$. Use of sustained-release preparations, particularly without appropriate increases in dosing intervals, coadministration of an over-the-counter, fixed-dose combination product without recognizing that it contains paracetamol, or supervision of medication administration by another child may also contribute to such errors.

Thirdly, reports of liver toxicity in paediatric patients have suggested a minimal, single paracetamol dose of $120-150 \mathrm{mg} / \mathrm{kg}$ body weight may be associated with hepatotoxicity ${ }^{3,4}$. In general, risks of developing toxic reactions to paracetamol appear to be lower in children than in adults ${ }^{5}$. But, in several cases of children with fulminant liver failure after multiple paracetamol doses, when serum paracetamol levels were tested, they were within or below the therapeutic range. Hence, accurate knowledge about the timing between the last dose and sampling time is important. The conventional toxicity normogram is neither designed for diagnosing nor guiding management of chronic toxicity ${ }^{6}$. 
